\title{
Effects of Culture Duration, Follicle Stimulating Hormone (FSH) Type, and Activin A Concentration on In Vitro Growth of Preantral Follicles and Maturation of Intrafollicular Oocytes
}

\author{
Jung Kyu Choi* \\ Received June 14, 2019 \\ Revised June 20, 2019 \\ Accepted June 25, 2019 \\ *Correspondence
Jung Kyu Choi
Department of Biotechnology, College of
Life and Applied Sciences, Yeungnam
University, 280 Daehak-ro, Gyeongsan
38541, Korea
Tel: +82-53-810-0353
Fax: +82-53-810-4655
E-mail: jungkyuc@ynu.ac.kr
ORCID
https://orcid.org/0000-0003-4544-3668
}

Department of Biotechnology, College of Life and Applied Sciences, Yeungnam University, Gyeongsan 38541, Korea

\begin{abstract}
The objective of this study was to establish an in vitro culture system for ovarian preantral follicles of B6D2F1. First, we optimized the in vitro preantral-follicle culture by culture duration, follicle stimulating hormone (FSH) type, and activin A concentration. Duration of in vitro culture for 9,11 , and 13 days was sufficient for the normal development of preantral follicles to antral follicles. Formation of cumulus celloocyte complex (COC) was induced by treatment with human chorionic gonadotropin (hCG; $2.5 \mathrm{IU} / \mathrm{mL}$ ) and epidermal growth factor (EGF; $5 \mathrm{ng} / \mathrm{mL}$ ). In addition, metaphase II (MII) oocytes formed during this in vitro culture of preantral follicles. In vitro preantralfollicle culture for 9 days showed higher rates of growth and maturation, thus yielding a greater number of antral follicles, and there were significant differences $(p<0.05)$ in the number of MII oocytes (that formed from these preantral follicles via differentiation) between the 9-day culture and 11-day or 13-day culture. The follicles cultured for 9 days contained a tightly packed well-defined COC, whereas in follicles cultured for 11 days, the COC was not well defined (spreading was observed in the culture dish); the follicles cultured for 13 days disintegrated and released the oocyte. Second, we compared the growth of the preantral follicles in vitro in the presence of various FSH types. There were no significant differences in the growth and maturation rates and in differentiation into MII oocytes during in vitro culture between preantral follicles supplemented with FSH from Merck and those supplemented with FSH from Sigma. To increase the efficiency of MII oocyte formation, the preantral follicles were cultured at different activin A concentrations (0 to $200 \mathrm{ng} / \mathrm{mL}$ ). The control follicles, which were not treated with activin $A$, showed the highest rate of differentiation into antral follicles and into MII oocytes among all the groups (0 to $200 \mathrm{ng} / \mathrm{mL}$ ). Therefore, activin A (50 to $200 \mathrm{ng} / \mathrm{mL}$ ) had a negative effect on oocyte maturation. Thus, in this study, we propose an in vitro system of preantral-follicle culture that can serve as a therapeutic strategy for fertility preservation of human oocytes for assisted reproductive medicine, for conservation of endangered species, and for creation of superior breeds.
\end{abstract}

Keywords: fertility preservation, in vitro culture, oocyte, preantral follicle 


\section{INTRODUCTION}

The ovarian follicle, which is composed of an egg cell that is surrounded by somatic cells, is the smallest structural or functional tissue unit within the ovary, and a single follicle can become a mature ovum through folliculogenesis, followed by ovulation. Although women are born with approximately one million primordial follicles, less than $1 \%$ of these follicles is used throughout a woman's lifetime, and the remaining $99 \%$ of the primordial follicles spontaneously degenerate (Broekmans et al., 2006; Woodruff et al., 2008; Barnett et al., 2009; Choi et al., 2013). If the follicles that spontaneously degenerate were cultured in vitro, many oocytes could be retrieved, and the reproductive ability of women could also be restored through assisted reproductive technology. In addition, cultivation of preantral follicles in vitro could help preserve the genetic profile of endangered species or livestock with preferred traits (Donnez et al., 2010; Wang et al., 2018). Although in vitro culture techniques for preantral follicles have been actively studied in mice (Eppig et al., 1996; Kim et al., 2009), the use of following hormones was recently discontinued: follicle-stimulating hormone (FSH), which is the most important hormone involved in the stimulation of preantral-follicle growth, and human chorionic gonadotropin (hCG), which is important for in vitro maturation of oocytes. Hence, there is a need to re-establish the standards for in vitro culture of preantral follicles. Therefore, here, we established an in vitro follicle culture system optimized by culture duration, FSH types, and activin A concentration; these parameters are important for in vitro growth of follicles and maturation of oocytes. The in vitro follicle culture system established in this study could be a useful alternative for ensuring the fertility of women, endangered species, and livestock.

\section{MATERIALS AND METHODS}

\section{Animals and materials}

8 week-old B6D2F1 mice exclusively were provided by Koatech (Pyeoungtack, Korea). All procedures for animal management, breeding, and surgery were conducted in accordance with the standards of the Research Institute of Yeungnam University. All procedures described herein were reviewed and approved by the Institutional Animal Care and Use Committee of Yeungnam University and were performed in accordance with Guiding Principles for the Care and Use of Laboratory Animals (IACUC 180115). Leibovitz L-15, lyophilized penicillin-streptomycin solution and a-MEM-glutamax medium and fetal bovine serum (FBS) were purchased from Gibco and Corning, respectively. Unless otherwise specifically noted, all other chemicals were purchased from Sigma.

\section{Isolation of Preantral Follicles}

Early preantral follicles (100-125 $\mu \mathrm{m})$ were isolated using mechanical method from ovaries of 8 -week old female B6B2F1 mice. The ovaries were placed in a $2 \mathrm{~mL}$ Leibovitz L-15 medium supplemented with $10 \%(\mathrm{v} / \mathrm{v})$ heat-inactivated $\mathrm{FBS}$ and $1 \%(\mathrm{v} / \mathrm{v})$ penicillin-streptomycin at $37^{\circ} \mathrm{C}$ in $5 \% \mathrm{CO}_{2}$ air. Preantral follicles were retrieved by using two $30 \mathrm{G}$ needles to mechanically break the extracellular matrix between follicles in the ovarian tissue.

\section{In vitro culture of preantral follicles}

Preantral follicle (100-125 $\mu \mathrm{m})$ with multiple layers of granulosa cells and an intrafollicular oocyte were collected without enzymatic digestion, and the retrieved follicles were placed singly in $10 \mu \mathrm{L}$ culture droplets and overlaid with washed mineral oil in $60 \times 15 \mathrm{~mm}$ Falcon plastic Petri dishes (Becton Dickinson, Franklin Lakes, NJ, USA). Preantral follicles were cultured at $37^{\circ} \mathrm{C}$ containing $5 \% \mathrm{CO}_{2}$ in air in ribonucleoside- and deoxyribonucleoside containing $\alpha$-MEM-glutamax medium supplemented with FBS (5\%,v/v), insulin, transferrin, and selenium (ITS) liquid medium (1\%, v/v), recombinant human FSH (100 $\mathrm{mIU} / \mathrm{mL}$ ) and $1 \%(\mathrm{v} / \mathrm{v})$ lyophilized penicillin-streptomycin solution was added. To establish preantral follicle culture in vitro, according to culture duration (9,11 and 13 days), FSH types [Gonal-f (Merck) to sigma], or activin A concentration $(0,50,100$ and $200 \mathrm{ng} / \mathrm{mL})$. On the following day, $10 \mu \mathrm{L}$ of fresh medium was added to each drop, and starting from day 3 , half the medium $(10 \mu \mathrm{L})$ was replaced with the fresh medium every other day.

\section{In vitro maturation of oocytes in antral follicles}

To retrieve mature oocytes, early secondary follicles were cultured for 9, 11, and 13 days, which then culture duration of 9 days were performed in FSH type and activing A group. Maturation of oocyte was triggered by exposure to hCG $(2.5 \mathrm{IU} / \mathrm{mL})$ and epidermal growth factor $(5 \mathrm{ng} / \mathrm{mL}$ ) for $17 \mathrm{~h}$ prior to the end of culture. Retrieved 
oocytes were freed from cumulus cells by mechanical pipetting in L-15 medium supplemented with $200 \mathrm{IU} / \mathrm{mL}$ hyaluronidase. Oocytes at the germinal vesicle (GV) stage were judged by the presence of a clear GV observed under a phase-contrast microscope; GV breakdown (GVBD) oocytes were judged by the disappearance of the clear GV and MII oocytes were judged by the disappearance of the clear GV and the appearance of a characteristic first polar body.

\section{Statistical analysis}

A generalized linear model (PROC-GLM) in a Statistical
Analysis System (SAS Institute Inc., NC, USA) program was used for the statistical analysis to determine the $p$-value between various treatments. The differences were taken as significant when the $p$-value was less than 0.05 .

\section{RESULTS AND DISCUSSION}

To establish the culture conditions for preantral follicles, we cultured preantral follicles for a period of 9 to 13 days to determine the influence of duration on their development. As shown in Table 1, the percentage of preantral follicles that developed into antral follicles was the great-

Table 1. The influence of extended culture of early secondary follicles for up to 13 days on developmental competence of intrafollicular oocytes

\begin{tabular}{|c|c|c|c|c|c|c|}
\hline $\begin{array}{l}\text { Culture } \\
\text { duration }\end{array}$ & $\begin{array}{c}\text { No. of } \\
\text { follicles cultured }\end{array}$ & $\begin{array}{c}\text { No. (\%) of } \\
\text { antral follicles }\end{array}$ & No. of COCs & \multicolumn{3}{|c|}{ No. $(\%)^{b}$ of oocytes at the stage of } \\
\hline 9 days & 96 & $76(79)$ & 76 & $5(6)$ & $59(78)$ & $12(16)^{c}$ \\
\hline 13 days & 85 & $20(23)$ & 20 & $8(40)$ & $11(55)$ & $1(5)^{d}$ \\
\hline
\end{tabular}

COCs, cumulus-oocyte complexes; GV, germinal vesicle; GVBD, germinal vesicle breakdown; MII, metaphase II.

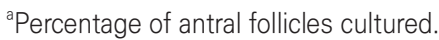

bercentage of GV, GVBD, or MII oocytes retrieved.

${ }^{\mathrm{c}, \mathrm{d}}$ Different superscripts for the same parameter (in the same column) indicate significant differences between the treatments, $p<0.05$.
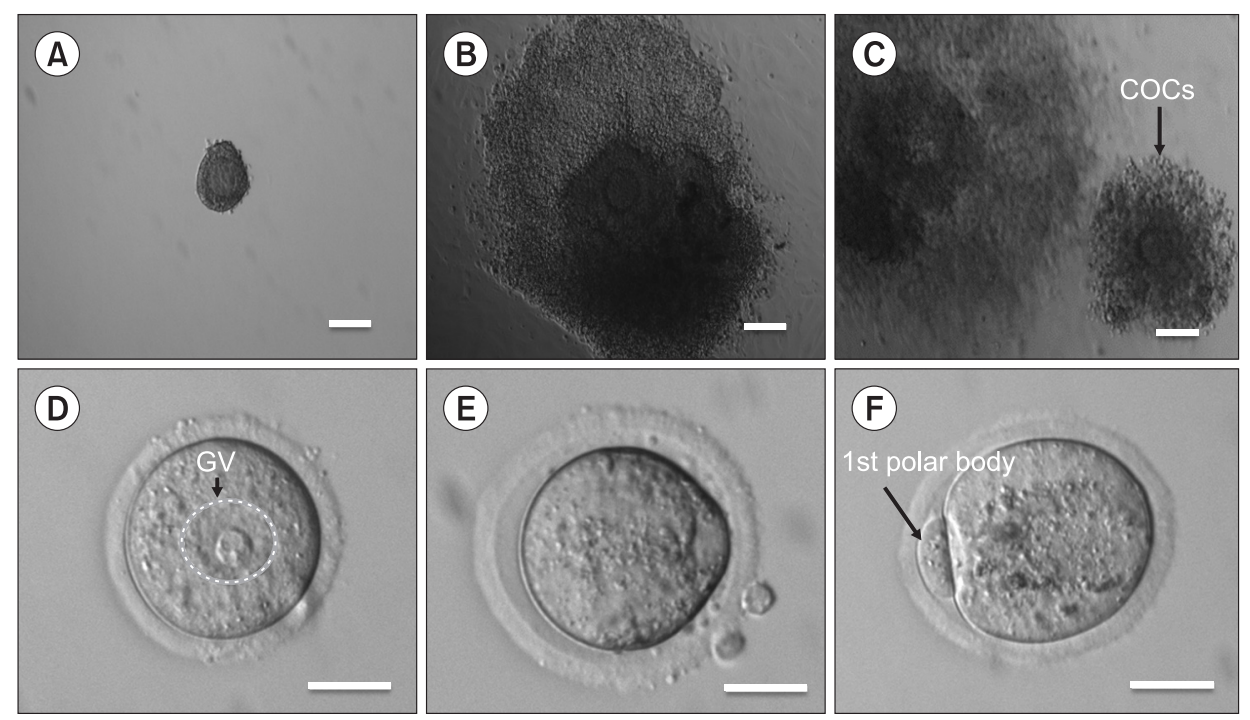

Fig. 1. Typical micrographs showing the development of a preantral follicle that was retrieved mechanically from an ovary of an 8-week-old mouse. The preantral follicle (A) under nonphysiological conditions developed to the antral stage (B). After further in vitro maturation, the cumulus-oocyte complex (COC; panel D) was released from the antral follicle. Cumulus cells in the COC (C) were removed using hyaluronidase to obtain a clean metaphase II (MII; F) oocyte containing the first polar body. Oocytes at the germinal vesicle (GV) stage were examined for the presence of a clear-cut GV (D) by examination under a phase contrast microscope; oocytes at the stage of GV breakdown (GVBD; panel E) were defined by the disappearance of the well-defined GV. Scale bars: $70 \mu \mathrm{m}$ (AC) or $35 \mu \mathrm{m}(\mathrm{D}-\mathrm{F})$. 
est after 9 days of culture (79\%) as compared to 11 days (68\%) and 13 days (23\%); the latter group showed the lowest proportion of preantral follicles that transformed into antral follicles. There was a statistically significant difference in the number of antral follicles formed after 9 or 11 days of culture compared to the 13 -day culture $(p<0.05)$. The follicles cultured for 9 days yielded a statistically significantly greater number of oocytes in metaphase II (MII) as compared to the preantral follicles cultured for 11 or 13 days ( $p<0.05$, Table 1). As illustrated in Fig.1, preantral follicles retrieved mechanically (Fig. 1A) were able to develop into antral follicles (Fig. 1B) in vitro. During in vitro maturation, we were able to track progression, i.e., the induction of transitions of the oocyte from its germinal-vesicle stage (GV; Fig. 1C) to the GV breakdown stage (GVBD; Fig. 1D) and to the stage of the first polar body in a metaphase II (MII) oocyte (Fig. 1E). Culturing of follicles for 9 days yielded the best outcome because a further increase in the incubation period had an adverse impact on the maturation of preantral follicles. The results depicted in Fig. 2 confirm the findings shown in Table 1, namely, that the follicles cultured for 9 days contained a COC with clear-cut morphology (Fig. 2A), whereas the follicles cultured for 11 days contained a COC that was not clearly defined and started to spread on the culture dish (Fig. 2B), and the 13-day culture produced degenerated follicles that released their oocyte (C). Follicle-stimulating hormone (FSH) is the most important stimulator of the growth of preantral follicles in ovaries. Since the original supply of FSH was discontinued, two types of FSH from Merck (GONAL-f) and from Sigma, which are commonly used in the clinical practice by OB-GYNs-were tested here to determine whether they are conducive to in vitro growth and maturation of oocytes in order to establish the in vitro culture system. As presented in Table 2, treatment with the FSH from Sigma, as compared to that from Merck, caused higher rates of antral-follicle formation (84\% vs. 81\%) and MII oocyte formation; however, these
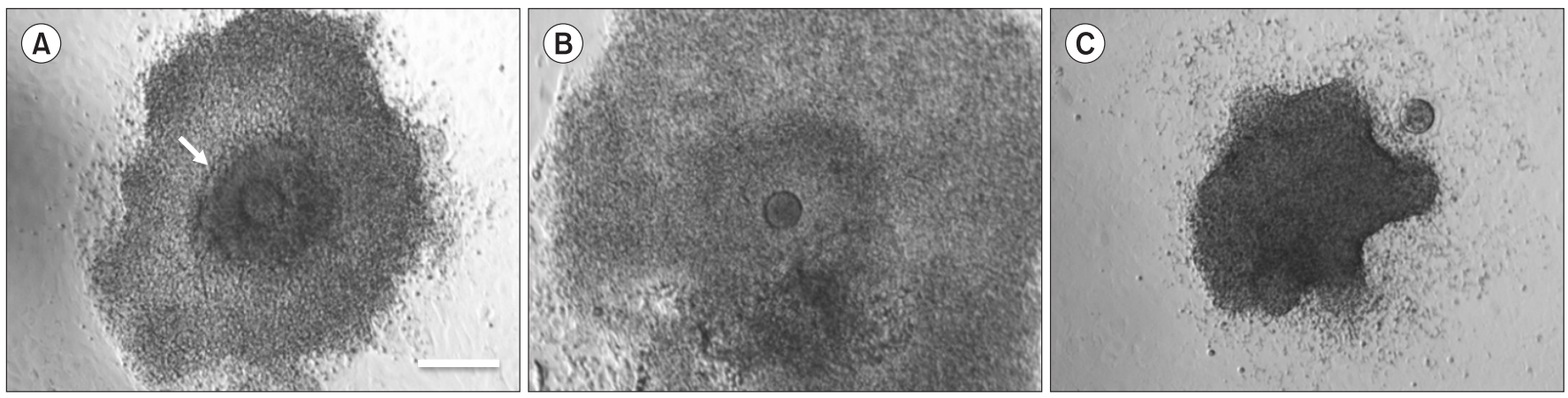

Fig. 2. Morphological features of follicles cultured in vitro for 9, 11, and 13 days. Follicles cultured for 9 (A), 11 (B), and 13 (C) days had different morphological features, namely, the follicles cultured for 9 days (A) developed to the antral stage and contained a clear-cut and tightly packed cumulus-oocyte complex (COC; arrow). By contrast, in the follicles cultured for 11 days, the COC spread on the culture dish and was not well-defined, whereas the follicles cultured for 13 days disintegrated and released the oocyte. Scale bar: $150 \mu \mathrm{m}$.

Table 2. The effect of the type of follicular-stimulating hormone (FSH) added to the culture of preantral follicles on the developmental competence of intrafollicular oocytes

\begin{tabular}{|c|c|c|c|c|c|c|}
\hline \multirow{2}{*}{ Type (FSH) } & \multirow{2}{*}{$\begin{array}{c}\text { No. of } \\
\text { follicles cultured }\end{array}$} & \multirow{2}{*}{$\begin{array}{c}\text { No. (\%) of } \\
\text { antral follicles }\end{array}$} & \multirow{2}{*}{ No. of COCs } & \multicolumn{3}{|c|}{ No. $(\%)^{b}$ of oocytes at the stage of } \\
\hline & & & & GV & GVBD & MII \\
\hline Merck & 96 & $78(81)$ & 78 & $16(17)$ & $58(74)$ & $4(5)$ \\
\hline Sigma & 92 & $77(84)$ & 77 & $13(17)$ & $55(71)$ & $9(12)$ \\
\hline
\end{tabular}

Merck (GONAL-f) is a human recombinant FSH.

Sigma FSH (cat. \# F4021) has been isolated from the human pituitary gland.

COCs, cumulus-oocyte complexes; GV, germinal vesicle; GVBD, germinal vesicle breakdown; MII, metaphase II.

aPercentage of antral follicles cultured.

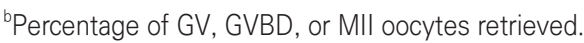


Table 3. The effect of the concentration of activin A added to the culture of preantral follicles on the developmental competence of intrafollicular oocytes

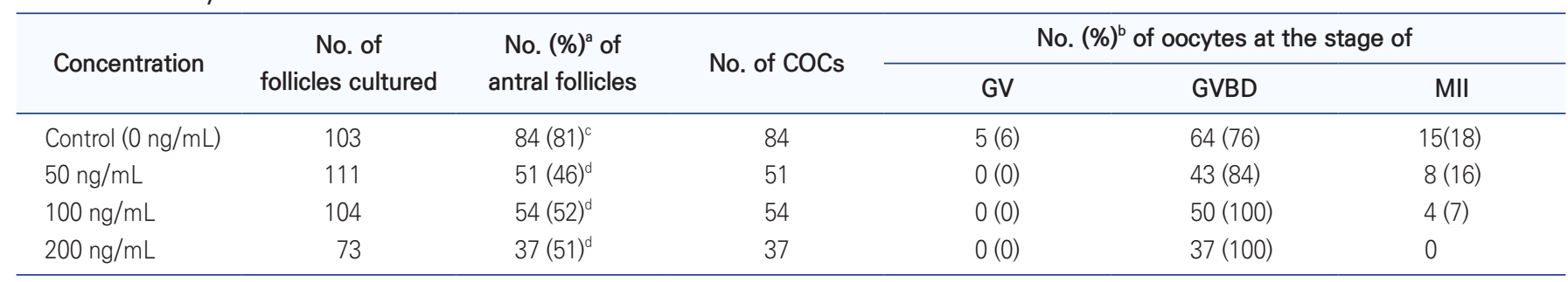

COCs, cumulus-oocyte complexes; GV, germinal vesicle; GVBD, germinal vesicle breakdown; MII, metaphase II.

aPercentage of antral follicles cultured.

${ }^{b}$ Percentage of GV, GVBD, or MII oocytes retrieved.

${ }^{\mathrm{c}, \mathrm{d}}$ Different superscripts for the same parameter indicate significant differences between the treatments, $p<0.05$.

differences were not statistically significant. Therefore, the Sigma brand of FSH was selected for the culture system.

Activin A belongs to the transforming growth factor $\beta$ (TGF- $\beta$ ) superfamily and reportedly affects granulosa cell proliferation during the antral-follicle stage of a primary follicle and can regulate growth, differentiation, and steroidogenesis in follicles ( $\mathrm{Li}$ et al., 1995). We, therefore, treated the preantral follicles with various concentrations ( 0 to $200 \mathrm{ng} / \mathrm{mL}$ ) of activin A to increase their growth and efficiency of transformation into a MII oocyte. Contrary to previous results, the group of follicles that was not treated with activin A showed the development of the largest number of antral follicles (81 antral follicles at 0 $\mathrm{ng} / \mathrm{mL}, 51$ at $50 \mathrm{ng} / \mathrm{mL}, 54$ at $100 \mathrm{ng} / \mathrm{mL}$, and 37 antral follicles at $200 \mathrm{ng} / \mathrm{mL}$ ), and the differences were statistically significant $(p<0.05$, Table 3$)$. Similarly, the group without activin A treatment showed the highest efficiency of maturation into the MII oocyte, but the differences in the rates of MII oocyte formation among the different groups were not statistically significant. Although previous studies have shown that activin A plays a pivotal role in the formation of follicles (Rabinovici et al., 1992; Li et al., 1995; Welt et al., 2002), its exact role has not yet been elucidated. Our results indicate that activin A exerts negative effects on preantral-follicle maturation; this outcome could be due to differences in the protocol or strains of mice among different studies.

Because the efficiency of MII oocyte development from preantral follicles is low, various growth factors were added to 2-dimensional (2D) culture of follicles. Nonetheless, we plan to develop an in vitro 3D culture system that can better reproduce the microenvironment of the ovary through bioengineering and tissue engineering (Desai et al., 2010; Belli et al., 2012; Yoon et al., 2019), possibly leading to higher rates of MII oocyte formation. On the basis of this 3D culture system, we expect to develop embryos via in vitro fertilization.

Because the use of the key hormones, FSH and hCG (which were traditionally employed in this field), has been discontinued, a new culture system for immature follicles was established here with optimized culture duration, FSH type, and activin A concentration. Thus, preantral follicles were cultured in vitro to induce MII oocyte formation with successful oogenesis. Our results suggest that the proposed in vitro system could help women undergoing fertility treatments as well as preserve the reproductive abilities of endangered species and genetic resources of livestock with desirable traits.

\section{CONFLICTS OF INTEREST}

No potential conflict of interest relevant to this article was reported.

\section{ACKNOWLEDGEMENTS}

This research was supported by the National Research Foundation of Korea (NRF) grant funded by the Korea government (MSIT) (2017R1C1B5076000) and by 2018 Yeungnam University Research Grant (218A580067).

\section{ORCID}

Jung Kyu Choi: https://orcid.org/0000-0003-4544-3668 


\section{REFERENCES}

Barnett KR, Schilling C, Greenfeld CR, Tomic D and Flaws JA. 2006. Ovarian follicle development and transgenic mouse models. Hum Reprod Update. 12:537-555.

Belli M, Vigone G, Merico V, Redi CA, Zuccotti M and Garagna, S. 2012. Towards a 3D culture of mouse ovarian follicles. Int J Dev Biol. 56:931-937.

Broekmans FJ, Soules MR, Fauser BC. 2009. Ovarian aging: mechanisms and clinical consequences. Endocrine Reviews. 30:465-493.

Choi JK, Agarwal P and He X. 2013. In vitro culture of early secondary preantral follicles in hanging drop of ovarian cellconditioned medium to obtain MII oocytes from outbred deer mice. Tissue Eng Part A. 19:2626-2637.

Desai N, Alex A, AbdelHafez F, Calabro A, Goldfarb J, Fleischman A, et al. 2010. Three-dimensional in vitro follicle growth: overview of culture models, biomaterials, design parameters and future directions. Reprod Biol Endocrinol. 8:119.

Donnez J and Dolmans MM. 2010. Cryopreservation and transplantation of ovarian tissue. Clin Obstet Gynecol. 53:787796.

Eppig J and O'Brien M. 1996. Development in vitro of mouse oocytes from primordial follicles. Biol Reprod. 54:197-207.
Jung-Ah Yoon and Jung Kyu Choi. 2019. In vitro growth of preantral follicle and maturation of intrafollicular oocyte from aged mice. J Anim Reprod Biotechnol. 34(1):35-39.

Kim IW, Gong SP, Yoo CR, Choi JH, Kim DY and Lim JM. 2009. Derivation of developmentally competent oocytes by the culture of preantral follicles retrieved from adult ovaries: maturation, blastocyst formation, and embryonic stem cell transformation. Fertil Steril. 92:1716-1724.

Li R, Phillips DM and Mather JP. (1995). Activin promotes ovarian follicle development in vitro. Endocrinology. 136:849856.

Rabinovici J, Spencer SJ, Doldi N, Goldsmith PC, Schwall R and Jaffe RB. 1992. Activin-A as an intraovarian modulator: actions, localization, and regulation of the intact dimer in human ovarian cells. J Clin Invest. 89:1528-1536.

Wang Y, Anazodo A and Logan S. 2019. Systematic review of fertility preservation patient decision aids for cancer patients. Psychooncology. 28:459-467.

Welt C, Sidis Y, Keutmann H and Schneyer A. 2002. Activins, inhibins, and follistatins: from endocrinology to signaling. A paradigm for the new millennium. Exp Biol Med. 227:724752.

Woodruff TK. 2008. Making eggs: is it now or later? Nature Medicine. 14:1190-1191. 